\title{
The Faint Young Sun and Faint Young Stars Paradox
}

\author{
Petrus C. Martens \\ Department of Physics \& Astronomy, \\ Georgia State University, \\ 25 Park Place, $6^{\text {th }}$ Floor, \\ Atlanta, GA 30303, USA \\ email: martens@astro.gsu.edu
}

\begin{abstract}
The purpose of this paper is to explore a resolution for the Faint Young Sun Paradox that has been mostly rejected by the community, namely the possibility of a somewhat more massive young Sun with a large mass loss rate sustained for two to three billion years. This would make the young Sun bright enough to keep both the terrestrial and Martian oceans from freezing, and thus resolve the paradox. It is found that a large and sustained mass loss is consistent with the well observed spin-down rate of Sun-like stars, and indeed may be required for it. It is concluded that a more massive young Sun must be considered a plausible hypothesis.
\end{abstract}

Keywords. Stars: late-type, stars: rotation, stars: mass loss, stars: evolution, solar wind

\section{Introduction}

The young Sun started its life on the main sequence with about $70 \%$ of the luminosity of what it has now according to standard stellar evolution theory. It is still a scientific riddle how, with such a faint Sun, the young Earth could be warm enough to host liquid water in its first couple of billion years. Yet geological evidence clearly indicates there have been warm oceans from very early on (Kasting 1989), and that these oceans were a key ingredient in the development of life.

This is called the Faint Young Sun Paradox. The paradox is even more compelling for the planet Mars which we know now to have been covered with oceans for periods of hundreds of millions of years in its early life, with only half of the incoming energy flux of sunlight of the Earth.

Stellar evolution simulations dictate his paradox and it therefore applies to all G stars, and less so for $\mathrm{K}$ and $\mathrm{M}$ stars that evolve much slower. In all cases the habitable zone around the star gradually moves outwards and planets that started out balmy are expected to end up scorched. Given that it took about four billion years on planet Earth for the development from single cell organisms to multi-cellular life - and since that is the only example of evolution we have - it is a reasonable assumption that the development of multicellular intelligent life takes a very long time in general, with most $\mathrm{G}$ star planets not spending enough time in the habitable zone.

This paradox has been known for a long time, and one of the first to hint at a solution was well known science popularizer Carl Sagan (Sagan \& Mullan 1972). Many solutions to the Faint Young Sun Paradox have been proposed over the years, and they come from very different fields. Fairly straightforward proposals are an enhanced greenhouse effect by carbon dioxide or methane, geothermal heath from an initially much warmer terrestrial core, a much smaller Earth albedo, life developing in a cold environment under a 200 meter thick ice sheet, a secular variation in the gravitational constant, etc. Most of these models have serious shortcomings: For example the greenhouse effect from methane 
appears to be self-limiting, and not enough CO2 is indicated by the geological record to justify a greatly enhanced greenhouse effect in the past (Kasting, 2004)

There is not enough space in a proceedings paper to review all the material discussed above, so I refer to a recent review by Feulner (2012) and a series of very enlightening presentations and papers by Dr. James Kasting (Kasting, Toon \& Pollack 1988; Kasting, 2004) on the many hypotheses proposed to resolve the Faint Young Sun Paradox.

Most of the solutions proposed for the Faint Young Sun Paradox apply to Earth alone; they do not explain the presence of liquid oceans on early Mars. Also, they are not solutions for the Faint Young Stars Paradox in general. A simpler solution has been proposed in that the early Sun was more massive and hence more luminous. This necessitates a massive, sustained solar wind for the first billions of years of the Sun's evolution, a condition that most in the community find implausible.

In the current paper I will explore the hypothesis of a more massive and hence much brighter young Sun in more detail, and investigate whether this leads to logical contradictions or can be ruled out by observations. I will find, surprisingly, that a more massive young Sun is not implausible at all, and links together what we know about stellar spin-down with simulations of the same. The results of this paper do not prove that the young Sun was more massive - we would need observations that demonstrate the sustained presence of a massive solar wind. But it does show that such a hypothesis cannot be ruled out at present, and consequently that the presence of oceans on early Mars may not be a conundrum, and that the habitable zones around solar analogs as well may remain in place for the billions of years it takes for multi-cellular and intelligent life to develop.

\section{Mass Loss and Luminosity}

A slightly more massive Sun would be significantly more luminous: In the solar portion of the Hertzsprung-Russel diagram luminosity scales with mass to the power 4 to 5 . If the Sun were more massive earlier on the Earth would be closer in as well: Because of conservation of it angular momentum the mean Sun-Earth distance varies as the inverse of the mass of the Sun, while the incoming radiation at the top of the Earth's atmosphere scales with the inverse of the square of that distance. Hence the amount of radiation the Earth receives varies as the mass of Sun to the power 6 to 7 . A $30 \%$ less luminous Sun at the Zero Age Main Sequence (ZAMS) at one solar mass could be compensated for by a mere 4 to $5 \%$ mass increase going back in time from the current Sun.

A sustained solar mass loss rate of roughly $10^{-11} \mathrm{M}_{\odot} \mathrm{yr}^{-1}$ is required to accomplish that. The current solar mass loss rate is estimated at $2-3 \times 10^{-14} \mathrm{M}_{\odot} \mathrm{yr}^{-1}$ for the fast wind and $10^{-15} \mathrm{M}_{\odot} \mathrm{yr}^{-1}$ for Coronal Mass Ejections (CMEs). Interestingly the mass loss from photon emission is twice as large, around $7 \times 10^{-14} \mathrm{M}_{\odot} \mathrm{yr}^{-1}$, but the latter contributes much less to angular momentum loss, because the photons are not forced to co-rotate with the magnetic field.

So the current mass loss rate, if extrapolated to the past, is insufficient to resolve the Faint Young Sun Paradox by a factor of 300 or more. Hence we must assume a much higher mass loss rate for the young Sun, sustained for several billions of years. Observations of some young solar-type stars indicate mass loss rates of roughly the right magnitude: e.g. 70 Opiuchi with a mass of 0.92 times that of the Sun, and an estimated age of 0.8 billion years, has a mass loss rate of $3 \times 10^{-12} \mathrm{M}_{\odot} \mathrm{yr}^{-1}$, and $\kappa^{-1}$ Cet (Do Nascimento et al. 2016) yields the same result from X-ray calibration.

A much more detailed analysis than the back-of-the-envelope calculation above, by Minton \& Malhotra (2007), narrows down the mass loss rate constraint further. Minton 
\& Malhotra calculate the solar mass and hence mass loss rate required to keep the radiative equilibrium temperature of the Earth's atmosphere at $273^{\circ} \mathrm{K}$, the freezing point of water, during solar evolution. It is plausibly assumed that the greenhouse effect will add about $15^{\circ} \mathrm{K}$ to that, as it does at present, to achieve the average atmospheric temperature we have now, that is favorable to life. The result of their analysis is, again, a required mass loss rate of about $10^{-11} \mathrm{M}_{\odot} \mathrm{yr}^{-1}$, but it only has to be sustained for the first 2.4 billion years.

The choice of maintaining the radiative equilibrium temperature at or above freezing is a rational one, because at a lower temperature planet Earth could flip to an equilibrium in which all of the surface is frozen over - snowball Earth - where the albedo is much higher, because of all the ice and snow. The geological record indicates that several "snowball Earth" episodes have occurred in Earth's history - in addition to the much more recent ice ages, where there is no full planetary ice coverage.

As an aside, but in response to an obvious question: How does the Earth's atmosphere escape from a "snowball Earth" state? The answer probably lies in the addition of CO2 to the atmosphere from volcanic eruptions. A slow but steady addition of $\mathrm{CO} 2$ by volcanism and no uptake of $\mathrm{CO} 2$ by the weathering of rocks and diffusion into the oceans - all covered by ice - will eventually create enough of a greenhouse effect to initiate melting at equatorial latitudes, after which, via various feedbacks, melting will proceed precipitously.

Minton \& Malhotra also point out that their model for "minimum mass loss", according to the simulations of Kasting (1991), maintains solar luminosity at a high enough level to keep the atmosphere of Mars above the freezing point for the first billion years of its history - when oceans are believed to have existed on Mars. So indeed a strong early solar wind can resolve the paradox for both Earth and Mars, no separate solutions are required, much to the liking of Father William of Occam.

\section{Stellar Spin Down and Mass Loss}

In this section I will relate stellar mass loss rates, which are hard to observe, with the much better known stellar spin down rates in order to verify whether these can be made consistent.

It is well known that Sun-like stars spin down from rotation periods of just a few days in their first billion years to several weeks in their mid-life, e.g. 26 days for the Sun at 4.5 billion years. The loss of angular momentum is usually ascribed to the torque applied by the stellar wind that co-rotates with the star near the surface and is forced to co-rotate roughly out to the Alfvén radius where the wind outflow velocity equals the Alfvén speed.

Weber \& Davis (1967) were the first to relate spin-down rates to a stellar wind model. Their model is of a purely radial magnetic field that changes polarity at the equator. Their key result that the torque applied by the wind on the star is to a good approximation given by

$$
T=\omega R_{A}^{2} \dot{M}
$$

where $\omega$ is the angular rotation rate of the star, $R_{A}$ the critical Alfvén radius where the wind outflow velocity equals the local Alfvén speed, and $\dot{M}$ is the stellar mass loss rate. The calculation of Weber \& Davis includes at factor $2 / 3$ on the right hand side resulting from the azimuthal integration of the torque, which I omit here for simplicity

Physically interpreted this means that the stellar wind torque is roughly equal to the angular momentum of a stellar wind forced to co-rotate up to $R_{A}$ and then let go, flowing out further preserving its angular momentum. The result of Eq. (3.1) follows from the requirement that the solution flows smoothly through the critical point in the defining 
equations, much like the requirement for the critical point in the thermally driven Parker wind.

The location of the critical Alfvén radius in the Weber \& Davis solution is 24 solar radii, while sophisticated numerical solutions (Keppens \& Goedbloed 2000, their Fig. 3) yield 7 to 14 stellar radii in the segment of their solution with open field lines - where the stellar wind comes from. Recent observations for the Sun (Velli, Tenerani \& DeForest 2016) also indicate that for the Sun the Alfvén radius is of the order of 12 radii out over the polar regions. So there is broad agreement between observations, theory and simulations here.

However, it turns out that the expression of Eq. (3.1) as defining the stellar wind torque is strongly dependent upon the geometry of the stellar coronal magnetic field. While the field in Weber \& Davis (1967) is purely radial (reversing at the equator), that in Keppens \& Goedbloed (2000) has a much more realistic "dead zone" over the equator, where the field is closed, while open field lines spread out from the poles. The dead zone in the simulations takes on a form very similar to observed solar helmet streamers, as observed during a solar eclipse.

The torque from the stellar wind in Keppens \& Goedbloed is a factor 15 to 60 smaller than that given by Eq. (3.1) (a factor 10 to 40 compared to Weber \& Davis), because of the difference in magnetic field topology. The same result had already been pointed out by Priest \& Pneuman (1974), based on the helmet streamer geometry of Pneuman \& Kopp (1971).

I will show now that this result has important implications both for the mass loss required for the Sun to slow down from its initial rotation rate at the ZAMS, to its current rate, and for the slow down of solar rotation in the remainder of the Sun's main sequence lifetime.

First we need to know the moment of inertia of a star to be able to estimate the slow down rate for a given stellar mass loss rate. The angular momentum of a star is given by

$$
L_{\star}=I_{\star} \omega=M_{\star}\left(\beta_{I} R_{\star}\right)^{2} \omega,
$$

where $L_{\star}$ is the angular momentum, $I_{\star}$ the moment of inertia, $\omega$ the rotation rate, $M_{\star}$ the stellar mass, $R_{\star}$ its radius, and $\beta_{I} R_{\star}$ the radius of gyration, with $\beta_{I}$ the gyration constant, i.e. the fraction of the radius for the arm in the moment of inertia. Stellar evolution codes show that the value of $\beta_{I}$ decreases a little after arrival of a star at the ZAMS, because of the production of Helium from lighter Hydrogen in the core (see Schrijver \& Zwaan 2000, their Sect. 13.1). Interestingly then we would expect Sun-like stars to slowly spin up as they evolve on the main sequence if it weren't for stellar mass loss. Later, as the star evolves towards its giant phase, the moment of inertia greatly increases of course, but that is of no concern here. The typical value of $\beta_{I}$ for a Sun-like star on the main sequence is of the order of 0.25 , the value I shall use from here on, and assumed to decrease much less than the rotation rate.

The decrease in angular momentum of a star as it evolves. i.e. $\dot{L_{\star}}$ is of course equal to the torque applied to it by the stellar wind, i.e.

$$
\dot{L_{\star}}=M_{\star}\left(\beta_{I} R_{\star}\right)^{2} \dot{\omega}=f \omega R_{A}^{2} \dot{M},
$$

where $f$ is the efficiency factor discussed above, determined by Keppens \& Goedbloed (2000) to range from $1 / 60$ to $1 / 15$. When we write the Alfvén radius $R_{A}$ as a multiple of the stellar radius, $\alpha_{A} R_{\star}$ a very simple expression results for the stellar mass loss that is required to produce the much better observed spin-down of late type stars after arriving 

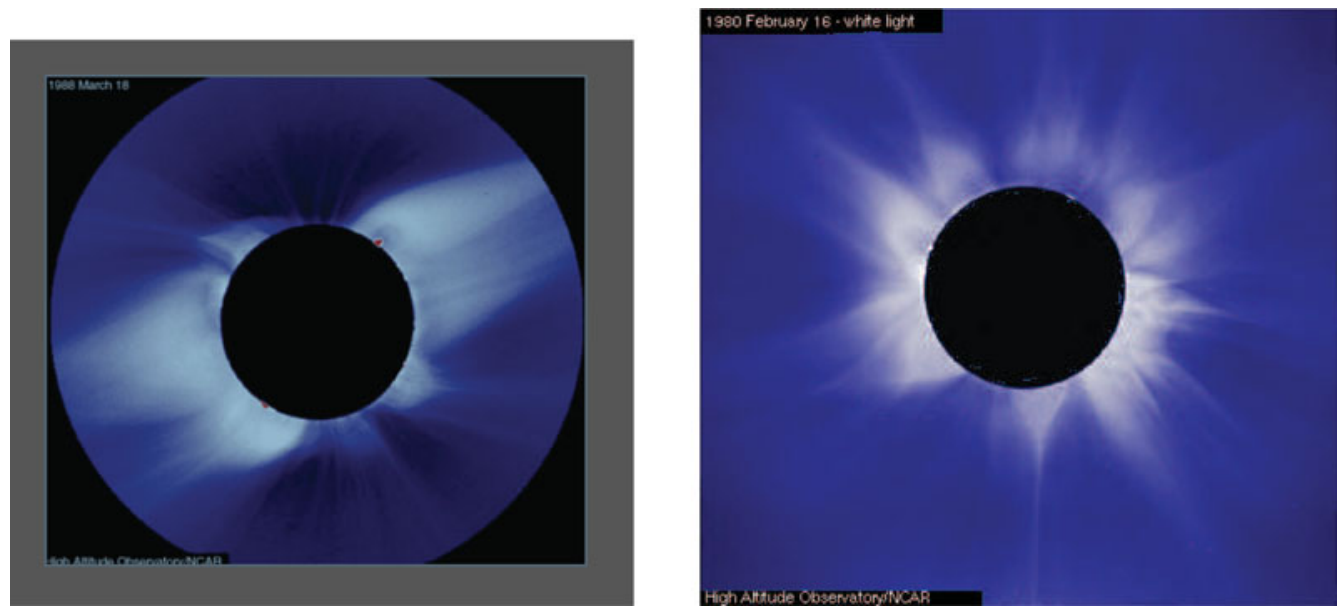

Figure 1. Helmet streamers observed during solar eclipses at solar minimum (left) and solar maximum (right). During maximum more streamers are present but their size is smaller than the ones at minimum.

on the main sequence,

$$
\dot{M}=\frac{\beta_{I}^{2}}{f \alpha_{A}^{2}} \frac{\dot{\omega}}{\omega} M_{\star} .
$$

The term $\frac{\dot{\omega}}{\omega}$ is simply the inverse of the e-folding time for the slow down in rotation of late type stars, which is of the order of 2-3 billion years, with not much variation between different stars (e.g. Nandy \& Martens, 2007). Above I have found $\beta_{I} \approx 0.25, \alpha_{A} \approx 10$, and $f \approx 1 / 30$. Inserting these values into Eq. (3.4) we derive our main result,

$$
\dot{M}_{\star}=-7.5 \times 10^{-12} M_{\star} y r^{-} 1 .
$$

This represents the mass loss required to explain the observed stellar spin down rate by magnetic breaking. This mass loss rate also equals the mass loss required to resolve the Faint Young Sun Paradox, as discussed in the previous section. Indeed a very large mass loss, sustained for several billion years, is not just a possibility, but it may very well be required to explain the spin down of Sun-like stars. The analysis above also demonstrates that at its current mass loss rate of $2-3 \times 10^{-14} \mathrm{M}_{\odot} \mathrm{yr}^{-1}$ our Sun will not slow down significantly for the remainder of its presence on the main sequence. This appears consistent with the observed rotation rates of older late type stars on the main sequence (Egeland, these proceedings).

\section{Discussion and Conclusions}

The current mass loss rate of $2-3 \times 10^{-14} \mathrm{M}_{\odot} \mathrm{yr}^{-1}$ is not sufficient to slow down the Sun from an initial rotation period of 4-5 days to its current value : That would require an Alfvén radius of 170 solar radii, beyond the orbit of Venus. One might argue that the young Sun most likely had a much stronger magnetic field, which would increase its Alfvén radius. However, the simulations of Keppens \& Goedbloed show that the slow down is not very sensitive to magnetic field strength: A stronger magnetic field is compensated by a larger dead zone, keeping the wind torque nearly constant.

Observations during solar eclipses even suggest a shrinking Alfvén radius with solar magnetic field. Fig. 1 shows juxtaposed helmet streamers observed during eclipses near solar minimum and solar maximum. The solar maximum image on the right shows more 
helmets, as expected, but of smaller size, with in particular the peak of the helmets closer to the solar surface. If, as in the simulations of Keppens \& Goedbloed, the peak of the helmets approximately coincides with the Alfvén radius at that position angle, the Alfvén radius of the Sun, in its current phase of evolution, is indeed smaller at higher activity levels.

I conclude that a high mass loss rate is a reasonable hypothesis to resolve both the Faint Young Sun and the Faint Young Stars Paradoxes. Observations of mass loss rates of late type stars in the first billions of years after their arrival on the ZAMS are needed to verify this hypothesis, as well as, if possible, investigations of remaining signatures of the early solar wind.

\section{References}

Kasting, J. F. 1989, Global and Planetary Change, 1, 83

Kasting, J. F. 1991, Icarus, 94, 1

Kasting, J. F. 2004, AGU Fall Meeting Abstracts

Kasting, J. F., Toon, O. B., \& Pollack, J. B. 1988, Scientific American, 258, 46

Keppens, R. \& Goedbloed, J. P. 2000, ApJ, 530, 1036

Minton, D. A. \& Malhotra, R. 2007, ApJ, 660, 1700

Nandy, D. \& Martens, P. C. H. 2007, Advances in Space Research, 40, 891

do Nascimento, J.-D., Jr., Vidotto, A. A., Petit, P., et al. 2016, ApJ Letters, 820, L15

Pneuman, G. W. \& Kopp, R. A. 1971, Sol. Phys., 18, 258

Priest, E. R. \& Pneuman, G. W. 1974, Sol. Phys., 34, 231

Sagan, C. \& Mullen, G. 1972, Science, 177, 52

Schrijver, C. J. \& Zwaan, C. 2000, Solar and stellar magnetic activity / Carolus J. Schrijver, Cornelius Zwaan. New York: Cambridge University Press, 2000. (Cambridge astrophysics series; 34)

Velli, M., Tenerani, A., \& DeForest, C. 2016, AAS/Solar Physics Division Meeting, 47, 402.05

Weber, E. J. \& Davis, L., Jr. 1967, ApJ, 148, 217 